\title{
$L$-series and their 2-adic valuations at $s=1$ attached to CM elliptic curves
}

\author{
by
}

Derong Qiu and Xianke Zhang (Beijing)

1. Introduction and statement of main results. Consider the family of elliptic curves:

$$
E: \quad y^{2}=x^{3}-D x
$$

defined over a number field $K$. Assume, first, $K=\mathbb{Q}$ is the rational field and $D \in \mathbb{Z}$ are rational integers (nevertheless, we will consider $K=\mathbb{Q}(\sqrt{-1})$ in the following). This family of elliptic curves $E$ have been studied thoroughly for a long time, having close relations with several problems of number theory. For example, they correlate intimately with the congruent number problem when $D$ is a square in $\mathbb{Z}$ (see [Tun]). Curves $E$ have complex multiplication by $\sqrt{-1}$, accordingly their complex $L$-series $L(E / \mathbb{Q}, s)$ (as curves over $\mathbb{Q}$ ) could be identified with the $L$-series $L(\psi, s)$ of Hecke characters $\psi$ (i.e. Grössencharakter) of the field $K=\mathbb{Q}(\sqrt{-1})$ attached to $E$. (Furthermore, when $E$ are considered as curves over the quadratic fields $K$ above, the $L$-series satisfy the relation

$$
L(E / K, s)=L(\psi, s) L(\bar{\psi}, s),
$$

where $\bar{\psi}$ is the dual of $\psi$.) The "conjecture of Birch and Swinnerton-Dyer" (or "BSD conjecture" for brevity) for an elliptic curve $E$ over $\mathbb{Q}$ asserts that the $L$-series $L(E / \mathbb{Q}, s)$ has a zero at $s=1$ of order $m$ equal to the rank $r$ of the Mordell group $E(\mathbb{Q})$, and gives a formula for the limit of $(s-1)^{-r} L(E / \mathbb{Q}, s)$ at $s=1$ involving arithmetic properties of $E$ (see e.g. [Sil1, p. 362]). In particular, for elliptic curves $E$ in (1.0) with complex multiplication, the BSD conjecture predicts that $L(E / K, 1)$ (after division by

2000 Mathematics Subject Classification: Primary 11G05; Secondary 11G15, 11G40, 12J20.

Key words and phrases: elliptic curve, complex multiplication, $L$-series and BSD conjecture, valuation. 
an appropriate period) is divisible by a certain power of 2 (see e.g. [Raz] and $[$ Tun]).

In 1997, C. L. Zhao considered the elliptic curves $E=E_{D}$ in (1.0) over the Gaussian field $\mathbb{Q}(\sqrt{-1})$ with the assumption that $D=\left(D_{0}\right)^{2}$ is a perfect square in $\mathbb{Z}[\sqrt{-1}]$, studying the 2 -adic valuation of $L\left(\psi_{D}, 1\right)$, the value at $s=1$ of the $L$-series of Hecke characters $\psi_{D}$ attached to $E_{D}$. (Actually, the value of the $L$-series should be first divided by an appropriate period $\Omega$; this normally will not be mentioned again in the following.) He gave a rigorous lower bound for the 2-adic valuation as well as a criterion of reaching this bound, and hence obtained nice results about congruent numbers and showed the BSD conjecture is true for some elliptic curves $E_{D}$ over $\mathbb{Q}$.

Here we study $E=E_{D}$ in the general case: $D$ is not necessarily a square. Consider the elliptic curves $E_{D}: y^{2}=x^{3}-D x$ over the Gaussian field $\mathbb{Q}(\sqrt{-1})$ with $D=\pi_{1} \ldots \pi_{n}$ and $D=\pi_{1}^{2} \ldots \pi_{r}^{2} \pi_{r+1} \ldots \pi_{n}$, where $\pi_{1}, \ldots, \pi_{n}$ are distinct Gaussian prime integers in $\mathbb{Z}[\sqrt{-1}]$. (In particular, when $r=n$, the second case turns out to be the case studied in [Zhao].) We will give a formula for the special values at $s=1$ of the Hecke $L$-series attached to $E=$ $E_{D}$ (expressed via the Weierstrass $\wp$-function), lower bounds for the 2-adic valuation of the values, and a criterion of reaching the bounds. These results develop the results for $E_{D}$ with $D=\left(D_{0}\right)^{2}$ square in the Gaussian field of [Zhao]. Moreover, our results are consistent with the predictions of the BSD conjecture. We will further study the elliptic curves $E: y^{2}=x^{3}-D$ having complex multiplication by $\sqrt{-3}$ over the field $\mathbb{Q}(\sqrt{-3})$ in a separate paper, giving results similar to the above but for the 3 -adic valuation.

Throughout the following, we put $I=\sqrt{-1}$, and let $K=\mathbb{Q}(\sqrt{-1})$ be the Gaussian number field, $O_{K}=\mathbb{Z}[\sqrt{-1}]$ the Gaussian integers (i.e. the ring of algebraic integers of $K), E_{D}: y^{2}=x^{3}-D x$ an elliptic curve defined over $K$ with complex multiplication by $O_{K}$. We let $\psi_{D}$ be the Hecke character of $K$ attached to $E_{D}$, and let $L\left(\bar{\psi}_{D}, s\right)$ denote the Hecke $L$-series of $\bar{\psi}_{D}$, the dual of $\psi_{D}$. (For the definition of such Hecke $L$-series attached to an elliptic curve, see [Sil2].)

(A) Consider $E_{D}: y^{2}=x^{3}-D x$ with $D=\pi_{1} \ldots \pi_{n}$, where $\pi_{k} \equiv 1$ $(\bmod 4)$ are distinct prime integers in $O_{K}(k=1, \ldots, n)$. Set $S=\left\{\pi_{1}, \ldots, \pi_{n}\right\}$. For any subset $T$ of $\{1, \ldots, n\}$, define

$$
D_{T}=\prod_{k \in T} \pi_{k}, \quad \widehat{D}_{T}=D / D_{T}
$$

and put $D_{\emptyset}=1$ when $T=\emptyset$. Let $\psi_{D_{T}}$ be the Hecke character (Grössencharakter) of the field $K$ attached to the elliptic curve $E_{D_{T}}: y^{2}=x^{3}-D_{T} x$, and let $L_{S}\left(\bar{\psi}_{D_{T}}, s\right)$ be the Hecke $L$-series of $\bar{\psi}_{D_{T}}$ (the dual of $\left.\psi_{D_{T}}\right)$ with all Euler factors at primes in $S$ omitted. We have the following formula for the 
special value $L_{S}\left(\bar{\psi}_{D_{T}}, 1\right)$ of the above $L$-series at $s=1$ expressed as a finite sum of the values of the Weierstrass $\wp$-function $\wp(z)$.

THEOREM 1. Let $\psi_{D_{T}}$ be the Hecke character of the Gaussian field $\mathbb{Q}(\sqrt{-1})$ attached to the elliptic curve $E_{D_{T}}: y^{2}=x^{3}-D_{T} x$, where $D_{T}$ is any factor of $D=\pi_{1} \ldots \pi_{n} \in \mathbb{Q}(\sqrt{-1})$ as above. Then we have the following formula for the value of L-series:

$$
\begin{aligned}
\frac{D}{\omega} \overline{\left(\frac{\theta}{D_{T}}\right)_{4} L_{S}\left(\bar{\psi}_{D_{T}}, 1\right)} \\
\quad=\frac{I}{2} \sum_{c \in \mathcal{C}}\left(\frac{c}{D_{T}}\right)_{4} \frac{1}{\wp(c \omega / D)-I}+\frac{1}{4} \sum_{c \in \mathcal{C}}\left(\frac{c}{D_{T}}\right)_{4}
\end{aligned}
$$

where $\theta=2+2 I,(-)_{4}$ is the quartic residue symbol, $\mathcal{C}$ is any complete set of representatives of $O_{K}$ modulo $D$ which are relatively prime to $D, L_{\omega}=\omega O_{K}$ is the period lattice of the elliptic curve $E_{1}: y^{2}=x^{3}-x$,

$$
\omega=\int_{1}^{\infty} \frac{d x}{\sqrt{x^{3}-x}}=2.6220575 \ldots
$$

$\wp(z)$ is the Weierstrass $\wp$-function associated to the lattice $L_{\omega}$ (i.e., $\wp(z)$ and its derivative $\wp^{\prime}(z)$ satisfy the equation $\left.\wp^{\prime}(z)^{2}=4 \wp(z)^{3}-4 \wp(z)\right)$.

REMARK 1.1. Formula (1.1) and its proof are developed from a famous formula and proof of Birch and Swinnerton-Dyer for elliptic curves over the rationals $\mathbb{Q}$ in [B-SD, Formula 3.14].

For any prime number $p$, we let $\mathbb{Q}_{p}$ be the completion of $\mathbb{Q}$ at the $p$-adic valuation, $\overline{\mathbb{Q}}$ and $\overline{\mathbb{Q}}_{p}$ the algebraic closures of $\mathbb{Q}$ and $\mathbb{Q}_{p}$ respectively; and let $v_{p}$ be the normalized $p$-adic exponential valuation of $\overline{\mathbb{Q}}_{p}$ (i.e. $v_{p}(p)=1$ ). Fix an isomorphic embedding $\overline{\mathbb{Q}} \hookrightarrow \overline{\mathbb{Q}}_{p}$. Then $v_{p}(\alpha)$ is defined for any algebraic number $\alpha$ in $\overline{\mathbb{Q}}$. The value $v_{p}(\alpha)$ for $\alpha \in \overline{\mathbb{Q}}$ depends on the choice of the embedding $\overline{\mathbb{Q}} \hookrightarrow \overline{\mathbb{Q}}_{p}$, but this does not affect our discussion in this paper. We will discuss the case $p=2$.

For any Gaussian integers $\alpha, \beta$ which are relatively prime, write $(\alpha / \beta)_{4}^{2}$ $=(\alpha / \beta)_{2}$ for short, and define $[\alpha / \beta]_{2}=\left(1-(\alpha / \beta)_{2}\right) / 2$. Then $[\alpha \gamma / \beta]_{2}=$ $[\alpha / \beta]_{2}+[\gamma / \beta]_{2}$ (regard $[-]_{2}$ as an $\mathbb{F}_{2}$-valued function, where $\mathbb{F}_{2}$ is the finite field with two elements). For $D=\pi_{1} \ldots \pi_{n}$ as above, put

$$
S^{*}(D)=\frac{I}{2} \sum_{c \in \mathcal{C}} \frac{1}{\wp(c \omega / D)-I} \sum_{T}\left(\frac{c}{D_{T}}\right)_{4} .
$$

We will show that $v_{2}\left(S^{*}(D)\right) \geq(n-1) / 2$ (see Lemma 2.4). Accordingly we define an $\mathbb{F}_{2}$-valued function $\varepsilon_{n}$ as follows $(n=n(D)$ is the number of 
distinct prime factors of $D)$ :

$$
\varepsilon_{n}(D)= \begin{cases}1 & \text { if } v_{2}\left(S^{*}(D)\right)=(n-1) / 2, \\ 0 & \text { if } v_{2}\left(S^{*}(D)\right)>(n-1) / 2 .\end{cases}
$$

Then for Gaussian prime integers $\pi, \pi_{k}$ congruent to 1 modulo $4(1 \leq$ $k \in \mathbb{Z}$ ) (and their products), we could define the $\mathbb{F}_{2}$-valued functions $s_{1}$ and $\delta_{n}(n=1,2, \ldots)$ inductively as follows:

$$
\begin{aligned}
s_{1}(\pi) & = \begin{cases}1 & \text { if } v_{2}(\pi-1)=2, \\
0 & \text { if } v_{2}(\pi-1)>2,\end{cases} \\
\delta_{1}(\pi) & =s_{1}(\pi)+\varepsilon_{1}(\pi), \\
\delta_{n}(D) & =\delta_{n}\left(\pi_{1}, \ldots, \pi_{n}\right) \\
& =\varepsilon_{n}(D)+\sum_{\emptyset \neq T \mp\{1, \ldots, n\}}\left(\prod_{k \notin T}\left[\frac{D_{T}}{\pi_{k}}\right]_{2}\right) \delta_{t}\left(D_{T}\right) \quad(n \geq 2),
\end{aligned}
$$

where $t=\sharp T$ is the cardinal of $T$.

TheOREM 2. Let $\psi_{D}$ be the Hecke character of $\mathbb{Q}(\sqrt{-1})$ attached to the elliptic curve $E_{D}: y^{2}=x^{3}-D x$, where $D=\pi_{1} \ldots \pi_{n}$ with $\pi_{k} \equiv 1$ $(\bmod 4)$ distinct Gaussian prime integers $(k=1, \ldots, n)$. Then for the 2-adic valuation of the values of the $L$-series $L\left(\bar{\psi}_{D}, s\right)$ at $s=1$ we have:

(i) $v_{2}\left(L\left(\bar{\psi}_{D}, 1\right) / \omega\right) \geq(n-1) / 2$;

(ii) Equality holds in (i) if and only if $\delta_{n}(D)=1$.

Theorem 3. Let $D= \pm p_{1} \ldots p_{m} \equiv 1(\bmod 4)$ with $p_{k} \not \equiv 5(\bmod 8)$ distinct positive rational prime numbers $(k=1, \ldots, m)$. If $\delta_{n}(D)=1$, then the first part of the BSD conjecture is true for the elliptic curve $E_{D}: y^{2}=$ $x^{3}-D x$, that is,

$$
\operatorname{rank}\left(E_{D}(\mathbb{Q})\right)=\operatorname{Ord}_{s=1}\left(L\left(E_{D} / \mathbb{Q}, s\right)\right)=0,
$$

where $n=n(D)$ is the number of distinct Gaussian prime factors of $D$.

(B) Consider the elliptic curves $E_{D}: y^{2}=x^{3}-D x$ with

$$
D=\pi_{1}^{2} \ldots \pi_{r}^{2} \pi_{r+1} \ldots \pi_{n},
$$

where $\pi_{k} \equiv 1(\bmod 4)$ are distinct prime integers in $\mathbb{Z}[\sqrt{-1}](k=1, \ldots, n)$. Let $\Delta=\pi_{1} \ldots \pi_{n}$ and $S=\left\{\pi_{1}, \ldots, \pi_{n}\right\}$. For any subset $T$ of $\{1, \ldots, n\}$, define

$$
D_{T}=\prod_{r \geq k \in T} \pi_{k}^{2} \prod_{r<j \in T} \pi_{j} .
$$

Let $L_{S}\left(\bar{\psi}_{D_{T}}, s\right)$ denote the Hecke $L$-series of $\bar{\psi}_{D_{T}}$ (omitting all Euler factors corresponding to primes in $S$ ), where $\psi_{D_{T}}$ is the Hecke character of $K=$ $\mathbb{Q}(\sqrt{-1})$ attached to $E_{D_{T}}: y^{2}=x^{3}-D_{T} x$. 
Theorem 4. For any factor $D_{T}$ of $D=\pi_{1}^{2} \ldots \pi_{r}^{2} \pi_{r+1} \ldots \pi_{n} \in \mathbb{Q}(\sqrt{-1})$ as above, we have

$$
\frac{\Delta}{\bar{\omega}\left(\frac{\theta}{D_{T}}\right)_{4}} L_{S}\left(\bar{\psi}_{D_{T}}, 1\right)=\frac{I}{2} \sum_{c \in \mathcal{C}}\left(\frac{c}{D_{T}}\right)_{4} \frac{1}{\wp(c \omega / \Delta)-I}+\frac{1}{4} \sum_{c \in \mathcal{C}}\left(\frac{c}{D_{T}}\right)_{4},
$$

where $\mathcal{C}$ is any complete set of representatives of $O_{K}$ modulo $\Delta$ relatively prime to $\Delta, \theta=2+2 I, L_{\omega}=\omega O_{K}, \omega$ and $\wp(z)$ are as in Theorem 1 .

TheOREM 5. Let $D=\pi_{1}^{2} \ldots \pi_{r}^{2} \pi_{r+1} \ldots \pi_{n}$, where $n, r$ are positive integers $(1 \leq r \leq n)$ (if $r=n$ then $\left.D=\pi_{1}^{2} \ldots \pi_{n}^{2}\right)$ and $\pi_{k} \equiv 1(\bmod 4)$ are distinct prime Gaussian integers $(k=1, \ldots, n)$. Then for the 2-adic valuation of the values of the $L$-series we have

$$
v_{2}\left(L\left(\bar{\psi}_{D}, 1\right) / \omega\right) \geq \frac{n}{2}-1,
$$

where $\psi_{D}$ is the Hecke character of $\mathbb{Q}(\sqrt{-1})$ attached to the elliptic curve $E_{D}: y^{2}=x^{3}-D x$.

2. Proofs of the theorems. We need the following results.

Proposition A. Let $E$ be an elliptic curve defined over an imaginary quadratic field $K$ with complex multiplication by $O_{K}$ (the ring of integers of $K), L=\Omega O_{K}$ be its period lattice, $\Omega \in \mathbb{C}^{\times}$a complex number, and $\phi$ be the Hecke character of $K$ attached to $E$. Assume $\mathfrak{g}$ is an integral ideal of $K, E_{\mathfrak{g}}$ is the group of $\mathfrak{g}$-divisible points on $E$. Let $\mathbf{B}$ be a set of integral ideals of $K$ relatively prime to $\mathfrak{g}$ such that

$$
\left\{\sigma_{b} \mid b \in \mathbf{B}\right\}=\operatorname{Gal}\left(K\left(E_{\mathfrak{g}}\right) / K\right), \quad \sigma_{b} \neq \sigma_{b^{\prime}} \quad \text { if } b \neq b^{\prime},
$$

where $\sigma_{b}=\left(\frac{K\left(E_{\mathfrak{g}}\right) / K}{b}\right)$ is the Artin symbol. Assume $\varrho \in \Omega K^{\times}$is a complex number such that $\varrho \Omega^{-1} O_{K}=\mathfrak{g}^{-1} \mathfrak{h}$ for some integral ideal $\mathfrak{h}$ of $K$ which is relatively prime to $\mathfrak{g}$. Then

$$
\left.\frac{\phi^{k}(\mathfrak{h})}{N(\mathfrak{h})^{k-s}} \cdot \frac{\bar{\varrho}^{k}}{|\varrho|^{2 s}} \cdot L_{\mathfrak{g}} \bar{\phi}^{k}, s\right)=\sum_{b \in \mathbf{B}} H_{k}(\phi(b) \varrho, 0, s, L)
$$

$(\operatorname{Re}(s)>1+k / 2)$, where $k$ is a positive integer and $N$ denotes the norm map from $K$ to $\mathbb{Q}$,

$$
\begin{gathered}
\left.L_{\mathfrak{g}} \bar{\phi}^{k}, s\right)=\prod_{\wp \nmid \mathfrak{g}}\left(1-\bar{\phi}^{k}(\wp) N(\wp)^{-s}\right)^{-1} \quad(\operatorname{Re}(s)>1+k / 2), \\
H_{k}(z, 0, s, L)=\sum^{\prime} \frac{(\bar{z}+\bar{\alpha})^{k}}{|z+\alpha|^{2 s}} \quad(\operatorname{Re}(s)>1+k / 2),
\end{gathered}
$$

here $\sum^{\prime}$ is taken over $\alpha \in L$ other than $-z$ if $z \in L$ (see [Go-Sch]). 
Lemma B. Let the elliptic curve E, field $K$, Hecke character $\phi$, and $\mathfrak{g}$ be as in Proposition A. If the conductor $f_{\phi}$ of $\phi$ divides $\mathfrak{g}$, then the ray class field of $K$ modulo $\mathfrak{g}$ is $K\left(E_{\mathfrak{g}}\right)$, the extension of $K$ obtained by adding the coordinates of all $\mathfrak{g}$-division points of $E$ to $K$ (see [Go-Sch]).

Now we consider Theorem 1 and let $K, E_{D}, D_{T}$ and $L_{S}\left(\bar{\psi}_{D_{T}}, s\right)$ be as there. Then by definition (see [B-SD], [Ire-Ro]) we have

LEMMA 2.1.

$$
\begin{aligned}
L_{S}\left(\bar{\psi}_{D_{T}}, s\right) & \begin{cases}L\left(\bar{\psi}_{D_{T}}, s\right) & \text { if } \prod_{\pi_{k} \in S} \pi_{k}=D_{T}, \\
L\left(\bar{\psi}_{D_{T}}, s\right) \prod_{\pi_{k} \mid \widehat{D}_{T}}\left(1-\left(\frac{D_{T}}{\pi_{k}}\right)_{4} \cdot \bar{\pi}_{k} /\left(\pi_{k} \bar{\pi}_{k}\right)^{s}\right) & \text { otherwise. }\end{cases}
\end{aligned}
$$

Proof of Theorem 1. Assume $L=\Omega O_{K}$ is the period lattice of $E_{D_{T}}: y^{2}=$ $x^{3}-D_{T} x$, where $\Omega=\alpha \omega, \alpha \in \mathbb{C}^{\times}$. (Obviously $\Omega=\omega / \sqrt[4]{D_{T}}$.) From [Bir-Ste] we know that the conductor of $\psi_{D_{T}}$ is $\left(\theta D_{T}\right)$. Now, in Proposition A, let $k=1, \varrho=\Omega /(\theta D), \mathfrak{g}=(\theta D), \mathfrak{h}=O_{K}$. We have

$$
\frac{\bar{\varrho}}{|\varrho|^{2 s}} L_{\mathfrak{g}}\left(\bar{\psi}_{D_{T}}, s\right)=\sum_{b \in \mathbf{B}} H_{1}\left(\psi_{D_{T}}(b) \varrho, 0, s, L\right) \quad(\operatorname{Re}(s)>3 / 2) .
$$

Since the conductor of $\psi_{D_{T}}$ is $\theta D_{T}$, and $\left(\theta D_{T}\right) \mid(\theta D)=\mathfrak{g}$, by Lemma B the ray class field of $K$ modulo $(\theta D)$ is $K\left(\left(E_{D_{T}}\right)_{(\theta D)}\right)$, the extension of $K$ obtained by adding the coordinates of $\theta D$-division points of $E_{D_{T}}$ to $K$. In particular we have the following isomorphism via the Artin map:

$$
\left(O_{K} /(\theta D)\right)^{\times} / \mu_{4} \cong \operatorname{Gal}\left(K\left(\left(E_{D_{T}}\right)_{(\theta D)}\right) / K\right),
$$

where $\mu_{4}$ is the group of quartic roots of unity, and $\mu_{4} \cong\left(O_{K} / \theta\right)^{\times}$. So we may take the set

$$
\mathbf{B}=\{(c \theta+D) \mid c \in \mathcal{C}\},
$$

where $\mathcal{C}$ is as in Theorem 1 , a set of representatives of $\left(O_{K} /(D)\right)^{\times}$; thus

$$
\frac{\bar{\varrho}}{|\varrho|^{2 s}} L_{\mathfrak{g}}\left(\bar{\psi}_{D_{T}}, s\right)=\sum_{c \in \mathcal{C}} H_{1}\left(\psi_{D_{T}}(c \theta+D) \varrho, 0, s, L\right) \quad(\operatorname{Re}(s)>3 / 2) .
$$

Note that the analytic continuation of $H_{1}(z, 0,1, L)$ can be given by the Eisenstein $E^{*}$-function (see [Zhao] or [We]):

$$
H_{1}(z, 0,1, L)=E_{0,1}^{*}(z, L)=E_{1}^{*}(z, L) .
$$

So by (2.3) we have 


$$
\frac{\theta D}{\Omega} L_{(\theta D)}\left(\bar{\psi}_{D_{T}}, 1\right)=\sum_{c \in \mathcal{C}} E_{1}^{*}\left(\psi_{D_{T}}(c \theta+D) \frac{\Omega}{\theta D}, \Omega O_{K}\right) .
$$

Since $D \equiv 1(\bmod 4)$, we get $c \theta+D \equiv 1(\bmod \theta)$ for any $c \in \mathcal{C}$. Thus by the definition of $\psi_{D_{T}}$ and quartic reciprocity law,

$$
\begin{aligned}
\psi_{D_{T}}(c \theta+D) & =\overline{\left(\frac{D_{T}}{c \theta+D}\right)_{4}}(c \theta+D)=\overline{\left(\frac{c \theta+D}{D_{T}}\right)_{4}}(c \theta+D) \\
& =\overline{\left(\frac{c \theta}{D_{T}}\right)_{4}}(c \theta+D) .
\end{aligned}
$$

Then by $(2.4)$ and the fact that $L_{(\theta D)}\left(\bar{\psi}_{D_{T}}, 1\right)=L_{S}\left(\bar{\psi}_{D_{T}}, 1\right)$, we have

$$
\frac{\theta D}{\alpha \omega} L_{S}\left(\bar{\psi}_{D_{T}}, 1\right)=\sum_{c \in \mathcal{C}} E_{1}^{*}\left(\left(\frac{c \omega}{D}+\frac{\omega}{\theta}\right) \alpha \overline{\left(\frac{c \theta}{D_{T}}\right)_{4}}, \alpha \omega O_{K}\right) .
$$

Put $\lambda=\alpha \overline{\left(\frac{c \theta}{D_{T}}\right)_{4}}$. Since $E_{1}^{*}(\lambda z, \lambda L)=\lambda^{-1} E_{1}^{*}(z, L)$, we have

$$
\begin{aligned}
E_{1}^{*}\left(\left(\frac{c \omega}{D}+\frac{\omega}{\theta}\right) \alpha \overline{\left(\frac{c \theta}{D_{T}}\right)_{4}}, \alpha \overline{\left(\frac{c \theta}{D_{T}}\right)_{4}} \omega O_{K}\right) \\
=\frac{1}{\alpha}\left(\frac{c \theta}{D_{T}}\right)_{4} E_{1}^{*}\left(\frac{c \omega}{D}+\frac{\omega}{\theta}, \omega O_{K}\right) .
\end{aligned}
$$

So by $(2.5)$,

$$
\frac{\theta D}{\omega} L_{S}\left(\bar{\psi}_{D_{T}}, 1\right)=\left(\frac{\theta}{D_{T}}\right) \sum_{4}\left(\frac{c}{D_{T}}\right)_{4} E_{1}^{*}\left(\frac{c \omega}{D}+\frac{\omega}{\theta}, \omega O_{K}\right) .
$$

For the period lattice $L_{\omega}=\omega O_{K}$ mentioned above, denote the corresponding Weierstrass $\wp$-function by $\wp\left(z, L_{\omega}\right)$ and the corresponding Weierstrass zetafunction by $\zeta\left(z, L_{\omega}\right)$. Then $\wp^{\prime}(z)^{2}=4 \wp(z)^{3}-4 \wp(z)$. So by results in [Go-Sch] we have

$$
\begin{aligned}
& \quad E_{1}^{*}\left(\frac{c \omega}{D}+\frac{\omega}{\theta}, \omega O_{K}\right) \\
& =\zeta\left(\frac{c \omega}{D}, L_{\omega}\right)+\zeta\left(\frac{\omega}{\theta}, L_{\omega}\right)+\frac{1}{2} \cdot \frac{\wp^{\prime}(c \omega / D)-(2-2 I)}{\wp(c \omega / D)-I}-\frac{\pi}{\omega} \overline{\left(\frac{c}{D}+\frac{1}{\theta}\right)} .
\end{aligned}
$$

We choose $\mathcal{C}$ in such a way that $c$ and $-c$ both are in $\mathcal{C}$. Obviously $\left(\frac{-c}{D_{T}}\right)_{4}=$ $\left(\frac{c}{D_{T}}\right)_{4}$. Since $\zeta\left(z, L_{\omega}\right)$ and $\wp^{\prime}\left(z, L_{\omega}\right)$ are odd functions, and $\wp\left(z, L_{\omega}\right)$ is even, by $(2.6)$ we have 


$$
\begin{aligned}
\frac{D}{\omega} & \left.\frac{\theta}{D_{T}}\right)_{4} L_{S}\left(\bar{\psi}_{D_{T}}, 1\right) \\
= & \frac{1}{\theta}\left\{\sum_{c \in \mathcal{C}}\left(\frac{c}{D_{T}}\right)_{4} \zeta\left(\frac{c \omega}{D}, L_{\omega}\right)-\frac{\pi}{\omega} \sum_{c \in \mathcal{C}}\left(\frac{c}{D_{T}}\right)_{4} \frac{\bar{c}}{\bar{D}}\right. \\
& \left.+\frac{1}{2} \sum_{c \in \mathcal{C}}\left(\frac{c}{D_{T}}\right)_{4} \frac{\wp^{\prime}(c \omega / D)}{\wp(c \omega / D)-I}-(1-I) \sum_{c \in \mathcal{C}}\left(\frac{c}{D_{T}}\right)_{4} \frac{1}{\wp(c \omega / D)-I}\right\} \\
& +\frac{1}{\theta} \sum_{c \in \mathcal{C}}\left(\frac{c}{D_{T}}\right)_{4}\left(\zeta\left(\frac{\omega}{\theta}, L_{\omega}\right)-\frac{\pi}{\omega \bar{\theta}}\right) \\
= & -\frac{1-I}{\theta} \sum_{c \in \mathcal{C}}\left(\frac{c}{D_{T}}\right)_{4} \frac{1}{\wp(c \omega / D)-I}+\frac{1}{\theta} \sum_{c \in \mathcal{C}}\left(\frac{c}{D_{T}}\right)_{4}\left(\zeta\left(\frac{\omega}{\theta}, L_{\omega}\right)-\frac{\pi}{\omega \bar{\theta}}\right) .
\end{aligned}
$$

That is,

$$
\begin{aligned}
& \frac{D}{\omega\left(\frac{\theta}{D_{T}}\right)_{4}} L_{S}\left(\bar{\psi}_{D_{T}}, 1\right) \\
= & \frac{I}{2} \sum_{c \in \mathcal{C}}\left(\frac{c}{D_{T}}\right)_{4} \frac{1}{\wp(c \omega / D)-I}+\frac{1}{\theta} \sum_{c \in \mathcal{C}}\left(\frac{c}{D_{T}}\right)_{4}\left(\zeta\left(\frac{\omega}{\theta}, L_{\omega}\right)-\frac{\pi}{\omega \bar{\theta}}\right) .
\end{aligned}
$$

By [Zhao] we know that

$$
\zeta\left(\frac{\omega}{\theta}, L_{\omega}\right)-\frac{\pi}{\omega \bar{\theta}}=\frac{\theta}{4}
$$

so

$$
\frac{D}{\bar{\omega}} \overline{\left(\frac{\theta}{D_{T}}\right)_{4}} L_{S}\left(\bar{\psi}_{D_{T}}, 1\right)=\frac{I}{2} \sum_{c \in \mathcal{C}}\left(\frac{c}{D_{T}}\right)_{4} \frac{1}{\wp(c \omega / D)-I}+\frac{1}{4} \sum_{c \in \mathcal{C}}\left(\frac{c}{D_{T}}\right)_{4} .
$$

This proves Theorem 1 .

LEMMA 2.2. We have

$$
\sum_{c \in \mathcal{C}}\left(\frac{c}{D_{T}}\right)_{4}= \begin{cases}\sharp \mathcal{C} & \text { if } T=\emptyset, \\ 0 & \text { if } T \neq \emptyset .\end{cases}
$$

Proof. Easy by the definition of quartic residue symbol.

Lemma 2.3. Let $D=\pi_{1} \ldots \pi_{n}$ where $\pi_{k} \equiv 1(\bmod 4)$ are distinct Gaussian prime $(k=1, \ldots, n)$. Let $c$ be any Gaussian integer relatively prime to $D$. Then

(1) $\sum_{T}\left(\frac{c}{D_{T}}\right)_{4}=\mu(1+I)^{t}$ or 0 , where $\mu \in\{ \pm 1, \pm I\}$, $t$ is an integer with $n \leq t \leq 2 n$.

(2) $\sum_{T}\left(\frac{c}{D_{T}}\right)_{4}=0$ if and only if $\left(\frac{c}{\pi_{k}}\right)_{4}=-1$ (for some $\left.k \in\{1, \ldots, n\}\right)$. 
(3) Suppose that $\left(\frac{c}{\pi_{k}}\right)_{4} \neq-1$ for any $k \in\{1, \ldots, n\}$. Then

$$
\begin{gathered}
\sum_{T}\left(\frac{c}{D_{T}}\right)_{4}=\mu(1+I)^{n+s}, \quad \text { where } \mu \text { is as in (1) above, } \\
s=\sharp\left\{\pi_{k}: \pi_{k} \mid D \text { and }\left(\frac{c}{\pi_{k}}\right)_{4}=1, k=1, \ldots, n\right\} .
\end{gathered}
$$

In particular,

$$
\begin{gathered}
\sum_{T}\left(\frac{c}{D_{T}}\right)_{4}=2^{n} \quad \text { if and only if }\left(\frac{c}{\pi_{1}}\right)_{4}=\ldots=\left(\frac{c}{\pi_{n}}\right)_{4}=1 \\
\sum_{T}\left(\frac{c}{D_{T}}\right)_{4}=\mu(1+I)^{n} \quad \text { if and only if }\left(\frac{c}{\pi_{k}}\right)_{4} \in\{I,-I\}, k=1, \ldots, n,
\end{gathered}
$$

where the sum $\sum_{T}$ is taken over all subsets $T$ of $\{1, \ldots, n\}$.

Proof of Lemma 2.3. In fact we have

$$
\sum_{T}\left(\frac{c}{D_{T}}\right)_{4}=\left(1+\left(\frac{c}{\pi_{1}}\right)_{4}\right) \ldots\left(1+\left(\frac{c}{\pi_{n}}\right)_{4}\right),
$$

from which the results could be deduced.

Lemma 2.4. $v_{2}\left(S^{*}(D)\right) \geq(n-1) / 2$.

Proof. By results of [Zhao] or [B-SD], we know that

$$
v_{2}\left(\wp\left(\frac{c \omega}{D}\right)-I\right)=\frac{3}{4}
$$

for any Gaussian integer $c$ relatively prime to $D$. By Lemma 2.3 we have

$$
v_{2}\left(\sum_{T}\left(\frac{c}{D_{T}}\right)_{4}\right)=v_{2}\left(\mu(1+I)^{t}\right)=\frac{t}{2} \geq \frac{n}{2} .
$$

(Here we regard $v_{2}(0)$ as $\infty$.) Thus by properties of valuation and our choice of $\mathcal{C}$ with the property $c,-c \in \mathcal{C}$, we have

$$
v_{2}\left(S^{*}(D)\right) \geq-\frac{3}{4}+\frac{n}{2} .
$$

Since $\pi_{k} \equiv 1(\bmod 4)(k=1, \ldots, n)$, it follows that

$$
N\left(D_{T}\right) \equiv N(D) \equiv 1(\bmod 8), \quad\left(\frac{I}{D_{T}}\right)_{4}=I^{\left(N\left(D_{T}\right)-1\right) / 4}= \pm 1 .
$$

Also

$$
\sharp\left(O_{K} /(D)\right)^{\times}=\sharp \mathcal{C}=\prod_{k=1}^{n}\left(N\left(\pi_{k}\right)-1\right) \equiv 0(\bmod 8),
$$


so we can choose $\mathcal{C}$ such that $\pm c, \pm I c \in \mathcal{C}$ (when $c \in \mathcal{C}$ ). Put

$$
V=\{c \in \mathcal{C}: c \equiv 1(\bmod \theta)\}, \quad V^{\prime}=V \cup I V .
$$

Then $\mathcal{C}=V^{\prime} \cup\left(-V^{\prime}\right)$. Since $I O_{K}=O_{K}$, we have $I L_{\omega}=I\left(\omega O_{K}\right)=\omega O_{K}=$ $L_{\omega}$. Thus by the definition of Weierstrass $\wp$-function,

$$
\begin{aligned}
\wp\left(I z, I L_{\omega}\right) & =\frac{1}{(I z)^{2}}+\sum_{\alpha \in I L_{\omega}}\left(\frac{1}{(I z-\alpha)^{2}}-\frac{1}{\alpha^{2}}\right) \\
& =\frac{1}{(I z)^{2}}+\sum_{\alpha^{\prime} \in L_{\omega}}\left(\frac{1}{\left(I z-I \alpha^{\prime}\right)^{2}}-\frac{1}{\left(I \alpha^{\prime}\right)^{2}}\right)=-\wp\left(z, L_{\omega}\right) .
\end{aligned}
$$

In particular,

$$
\begin{aligned}
& \wp\left(\frac{I c \omega}{D}, L_{\omega}\right)=-\wp\left(\frac{c \omega}{D}, L_{\omega}\right), \\
& S^{*}(D)= \frac{I}{2} \sum_{c \in \mathcal{C}} \frac{1}{\wp(c \omega / D)-I} \sum_{T}\left(\frac{c}{D_{T}}\right)_{4} \\
&= I \sum_{c \in V^{\prime}} \frac{1}{\wp(c \omega / D)-I} \sum_{T}\left(\frac{c}{D_{T}}\right)_{4} \\
&= I \sum_{c \in V}\left[\frac{1}{\wp(c \omega / D)-I} \sum_{T}\left(\frac{c}{D_{T}}\right)_{4}+\frac{1}{\wp(I c \omega / D)-I} \sum_{T}\left(\frac{I c}{D_{T}}\right)_{4}\right] \\
&= I \sum_{c \in V}\left[\sum_{T}\left(\frac{1}{\wp(c \omega / D)-I}-\left(\frac{I}{D_{T}}\right)_{4} \frac{1}{\wp(c \omega / D)+I}\right)\left(\frac{c}{D_{T}}\right)_{4}\right] \\
&= I \sum_{c \in V} \frac{2 B}{(\wp(c \omega / D))^{2}+1} \sum_{T}\left(\frac{c}{D_{T}}\right)_{4},
\end{aligned}
$$

where $B=I$ or $\wp(c \omega / D)$.

Note that $v_{2}(\wp(c \omega / D)-I)=3 / 4$, so $v_{2}(\wp(c \omega / D)+I)=3 / 4$. Hence

$$
\begin{gathered}
v_{2}\left(\left(\wp\left(\frac{c \omega}{D}\right)\right)^{2}+1\right)=v_{2}\left(\wp\left(\frac{c \omega}{D}\right)-I\right)+v_{2}\left(\wp\left(\frac{c \omega}{D}\right)+I\right)=\frac{3}{4}+\frac{3}{4}=\frac{3}{2}, \\
v_{2}\left(\frac{2 B}{(\wp(c \omega / D))^{2}+1}\right)=1-\frac{3}{2}=-\frac{1}{2}
\end{gathered}
$$

(and obviously we have $v_{2}(B)=0$ ). Therefore

$$
v_{2}\left(S^{*}(D)\right) \geq-\frac{1}{2}+v_{2}\left(\sum_{T}\left(\frac{c}{D_{T}}\right)_{4}\right) \geq-\frac{1}{2}+\frac{n}{2}=\frac{n-1}{2} .
$$

This proves Lemma 2.4 . 
Proof of Theorem 2. First let us prove

$$
v_{2}\left(L\left(\bar{\psi}_{D}, 1\right) / \omega\right) \geq(n-1) / 2 .
$$

Taking sums of both sides of formula (1.1) over subsets $T$ of $\{1, \ldots, n\}$, we have

$$
\begin{aligned}
\sum_{T} \frac{D}{\bar{\omega}} \overline{\left(\frac{\theta}{D_{T}}\right)_{4}} L_{S}\left(\bar{\psi}_{D_{T}}, 1\right) \\
=\frac{I}{2} \sum_{T} \sum_{c \in \mathcal{C}}\left(\frac{c}{D_{T}}\right)_{4} \frac{1}{\wp(c \omega / D)-I}+\frac{1}{4} \sum_{T} \sum_{c \in \mathcal{C}}\left(\frac{c}{D_{T}}\right)_{4} .
\end{aligned}
$$

So by Lemma 2.2 and (1.2), we obtain

$$
\begin{gathered}
\sum_{T} \frac{D}{\omega} \overline{\left(\frac{\theta}{D_{T}}\right)_{4}} L_{S}\left(\bar{\psi}_{D_{T}}, 1\right)=S^{*}(D)+\frac{\sharp \mathcal{C}}{4}, \\
v_{2}\left(\frac{\sharp \mathcal{C}}{4}\right)=v_{2}\left(\frac{\prod_{k=1}^{n}\left(\pi_{k} \bar{\pi}_{k}-1\right)}{4}\right) \geq 3 n-2 \geq n,
\end{gathered}
$$

and by Lemma 2.4 we have

$$
v_{2}\left(\sum_{T} \frac{D}{\omega} \overline{\left(\frac{\theta}{D_{T}}\right)_{4}} L_{S}\left(\bar{\psi}_{D_{T}}, 1\right)\right) \geq \frac{n-1}{2} .
$$

By Lemma 2.1 we know that $L_{S}\left(\bar{\psi}_{D_{T}}, 1\right)=L\left(\bar{\psi}_{D}, 1\right)$ when $T=\{1, \ldots, n\}$; and when $T=\emptyset$ we have

$L_{S}\left(\bar{\psi}_{D_{T}}, 1\right)=L_{S}\left(\bar{\psi}_{1}, 1\right)=L\left(\bar{\psi}_{1}, 1\right) \prod_{k=1}^{n}\left(1-\frac{\bar{\pi}_{k}}{\pi_{k} \bar{\pi}_{k}}\right)=L\left(\bar{\psi}_{1}, 1\right) \prod_{k=1}^{n}\left(1-\frac{1}{\pi_{k}}\right)$.

By [B-SD] or [Zhao] we know that $L\left(\bar{\psi}_{1}, 1\right)=\omega / 4$, so

$$
\begin{gathered}
L_{S}\left(\bar{\psi}_{1}, 1\right)=\frac{\omega}{4} \prod_{k=1}^{n}\left(1-\frac{1}{\pi_{k}}\right), \\
v_{2}\left(L_{S}\left(\bar{\psi}_{1}, 1\right) / \omega\right)=v_{2}\left(\frac{1}{4} \prod_{k=1}^{n}\left(1-\frac{1}{\pi_{k}}\right)\right) \geq 2 n-2 \quad\left(\text { since } v_{2}\left(\pi_{k}-1\right) \geq 2\right) .
\end{gathered}
$$

Now we use induction on $n$ to prove our assertion $v_{2}\left(L\left(\bar{\psi}_{D}, 1\right) / \omega\right) \geq(n-1) / 2$. If $n=1$, then $D=\pi_{1}, L_{S}\left(\bar{\psi}_{1}, 1\right)=(\omega / 4) \cdot\left(\pi_{1}-1\right) / \pi_{1}$. Since $\pi_{1} \equiv 1(\bmod 4)$, we get $v_{2}\left(L_{S}\left(\bar{\psi}_{1}, 1\right) / \omega\right) \geq 0$. By the above analysis we have

$$
v_{2}\left(\frac{\pi_{1}}{\omega}\left(\frac{\theta}{1}\right)_{4} L_{S}\left(\bar{\psi}_{1}, 1\right)+\frac{\pi_{1}}{\omega} \overline{\left(\frac{\theta}{\pi_{1}}\right)_{4}} L_{S}\left(\bar{\psi}_{\pi_{1}}, 1\right)\right) \geq \frac{1-1}{2}=0 .
$$

Therefore

$$
v_{2}\left(L\left(\bar{\psi}_{\pi_{1}}, 1\right) / \omega\right)=v_{2}\left(L_{S}\left(\bar{\psi}_{\pi_{1}}, 1\right) / \omega\right) \geq 0 .
$$


Now assume our assertion is true for $1, \ldots, n-1$, and consider $D=\pi_{1} \ldots \pi_{n}$. For any subset $T$ of $\{1, \ldots, n\}$, set $t=t(T)=\sharp T$. By Lemma 2.1,

$$
\frac{D}{\bar{\omega}} \overline{\left(\frac{\theta}{D_{T}}\right)_{4}} L_{S}\left(\bar{\psi}_{D_{T}}, 1\right)=\frac{D}{\omega} \overline{\left(\frac{\theta}{D_{T}}\right)_{4}} L\left(\bar{\psi}_{D_{T}}, 1\right) \prod_{\pi_{k} \mid \widehat{D}_{T}}\left(1-\left(\frac{D_{T}}{\pi_{k}}\right)_{4} \frac{1}{\pi_{k}}\right) .
$$

Since $\left(D_{T} / \pi_{k}\right)_{4}= \pm 1, \pm I$, we get

$$
1-\left(\frac{D_{T}}{\pi_{k}}\right)_{4} \frac{1}{\pi_{k}}=\frac{\pi_{k}-\mu}{\pi_{k}}, \quad \mu \in\{ \pm 1, \pm I\} .
$$

Note that $\pi_{k} \equiv 1(\bmod 4)$, so $v_{2}\left(\pi_{k}-\mu\right) \geq 1 / 2$; moreover equality holds if and only if $\left(D_{T} / \pi_{k}\right)_{4}^{2}=-1$. Thus when $T$ is non-trivial (i.e. $1 \leq t<n$ ), by our inductive assumption,

$$
\begin{array}{r}
v_{2}\left(\frac{D \bar{\omega}\left(\frac{\theta}{D_{T}}\right)_{4}}{L_{S}}\left(\bar{\psi}_{D_{T}}, 1\right)\right)=v_{2}\left(L\left(\bar{\psi}_{D_{T}}, 1\right) / \omega\right)+\sum_{\pi_{k} \mid \widehat{D}_{T}} v_{2}\left(1-\left(\frac{D_{T}}{\pi_{k}}\right)_{4} \frac{1}{\pi_{k}}\right) \\
\geq \frac{t-1}{2}+\frac{1}{2} \cdot \sharp\left\{\pi_{k}: \pi_{k} \mid \widehat{D}_{T}\right\}=\frac{t-1}{2}+\frac{n-t}{2}=\frac{n-1}{2} .
\end{array}
$$

Also when $T=\emptyset$ we have

$$
L_{S}\left(\bar{\psi}_{D_{T}}, 1\right)=L_{S}\left(\bar{\psi}_{1}, 1\right)=L\left(\bar{\psi}_{1}, 1\right) \prod_{k=1}^{n}\left(1-\frac{1}{\pi_{k}}\right)=\frac{\omega}{4} \prod_{k=1}^{n}\left(1-\frac{1}{\pi_{k}}\right),
$$

therefore

$$
\begin{aligned}
v_{2}\left(L_{S}\left(\bar{\psi}_{1}, 1\right) / \omega\right) \geq & 2 n-2 \geq(n-1) / 2, \\
v_{2}\left(L\left(\bar{\psi}_{D}, 1\right) / \omega\right)= & v_{2}\left(\frac{D}{\omega} \overline{\left(\frac{\theta}{D}\right)_{4}} L\left(\bar{\psi}_{D}, 1\right)\right) \\
= & v_{2}\left(\sum_{T} \frac{D}{\omega} \overline{\left(\frac{\theta}{D_{T}}\right)_{4}} L_{S}\left(\bar{\psi}_{D_{T}}, 1\right)\right. \\
& \left.-\sum_{\emptyset \neq T \varsubsetneqq\{1, \ldots, n\}} \frac{D}{\omega} \overline{\left(\frac{\theta}{D_{T}}\right)_{4}} L_{S}\left(\bar{\psi}_{D_{T}}, 1\right)-\frac{D}{\omega} L_{S}\left(\bar{\psi}_{1}, 1\right)\right) \\
\geq & (n-1) / 2 .
\end{aligned}
$$

Thus we have proved our assertion for any positive integer $n$.

Now we consider the condition for equality to hold, using also induction on $n$. If $n=1$, then $D=\pi_{1}$, and by (2.9) we obtain

$$
\frac{\pi_{1}}{\omega} \overline{\left(\frac{\theta}{1}\right)_{4}} L_{\pi_{1}}\left(\bar{\psi}_{1}, 1\right)+\frac{\pi_{1}}{\omega} \overline{\left(\frac{\theta}{\pi_{1}}\right)_{4}} L_{\pi_{1}}\left(\bar{\psi}_{\pi_{1}}, 1\right)=S^{*}\left(\pi_{1}\right)+\frac{\pi_{1} \bar{\pi}_{1}-1}{4},
$$


that is,

$$
\frac{1}{4}\left(\pi_{1}-1\right)+\frac{\pi_{1}}{\omega} \overline{\left(\frac{\theta}{\pi_{1}}\right)_{4}} L\left(\bar{\psi}_{\pi_{1}}, 1\right)=S^{*}\left(\pi_{1}\right)+\frac{\pi_{1} \bar{\pi}_{1}-1}{4} .
$$

Since

$$
v_{2}\left(\frac{\pi_{1} \bar{\pi}_{1}-1}{4}\right)=v_{2}\left(\pi_{1} \bar{\pi}_{1}-1\right)-2 \geq 1, \quad v_{2}\left(S^{*}\left(\pi_{1}\right)\right) \geq \frac{1-1}{2}=0
$$

(Lemma 2.4), the equality

$$
\begin{aligned}
v_{2}\left(L\left(\bar{\psi}_{\pi_{1}}, 1\right) / \omega\right) & =v_{2}\left(\frac{\pi_{1}}{\omega} \overline{\left(\frac{\theta}{\pi_{1}}\right)_{4}} L_{\pi_{1}}\left(\bar{\psi}_{\pi_{1}}, 1\right)\right) \\
& =v_{2}\left(S^{*}\left(\pi_{1}\right)+\frac{\pi_{1} \bar{\pi}_{1}-1}{4}-\frac{1}{4}\left(\pi_{1}-1\right)\right)=0
\end{aligned}
$$

holds if and only if one of the following conditions is true:

(1) $v_{2}\left(\pi_{1}-1\right)=2$ when $v_{2}\left(S^{*}\left(\pi_{1}\right)\right)>0$;

(2) $v_{2}\left(\pi_{1}-1\right)>2$ when $v_{2}\left(S^{*}\left(\pi_{1}\right)\right)=0$.

Thus

$v_{2}\left(L\left(\bar{\psi}_{\pi_{1}}, 1\right) / \omega\right)=0 \quad$ if and only if $\quad \delta_{1}\left(\pi_{1}\right)=s_{1}\left(\pi_{1}\right)+\varepsilon_{1}\left(\pi_{1}\right)=1$.

Assume our result is true for $1, \ldots, n-1$, and let $D=\pi_{1} \ldots \pi_{n}$. When $T=\emptyset$, we have

$$
\begin{aligned}
& \frac{D}{\omega} \overline{\left(\frac{\theta}{D_{T}}\right)_{4}} L_{S}\left(\bar{\psi}_{D_{T}}, 1\right)=\frac{D}{\omega} \overline{\left(\frac{\theta}{D_{T}}\right)_{4}} L_{D}\left(\bar{\psi}_{1}, 1\right)=\frac{D}{\omega} L\left(\bar{\psi}_{1}, 1\right) \prod_{k=1}^{n}\left(1-\frac{1}{\pi_{k}}\right) \text {, } \\
& v_{2}\left(\frac{D}{\omega} \overline{\left(\frac{\theta}{D_{T}}\right)_{4}} L_{S}\left(\bar{\psi}_{D_{T}}, 1\right)\right)=v_{2}\left(L\left(\bar{\psi}_{1}, 1\right) / \omega\right)+\sum_{k=1}^{n} v_{2}\left(\pi_{k}-1\right) \\
& =v_{2}(1 / 4)+\sum_{k=1}^{n} v_{2}\left(\pi_{k}-1\right) \geq 2 n-2 \geq(n-1) / 2 .
\end{aligned}
$$

When $\emptyset \neq T \varsubsetneqq\{1, \ldots, n\}$, we have

$$
\begin{aligned}
v_{2}\left(\frac{D}{\omega} \overline{\left.\left(\frac{\theta}{D_{T}}\right)_{4} L_{S}\left(\bar{\psi}_{D_{T}}, 1\right)\right)}=v_{2}\left(L_{S}\left(\bar{\psi}_{D_{T}}, 1\right) / \omega\right)\right. \\
=v_{2}\left(\frac{L\left(\bar{\psi}_{D_{T}}, 1\right)}{\omega} \prod_{\pi_{k} \mid \widehat{D}_{T}}\left(1-\left(\frac{D_{T}}{\pi_{k}}\right)_{4} \frac{1}{\pi_{k}}\right)\right) \\
=v_{2}\left(L\left(\bar{\psi}_{D_{T}}, 1\right) / \omega\right)+\sum_{\pi_{k} \mid \widehat{D}_{T}} v_{2}\left(1-\left(\frac{D_{T}}{\pi_{k}}\right)_{4} \frac{1}{\pi_{k}}\right) .
\end{aligned}
$$


Since $\left(D_{T} / \pi_{k}\right)_{4}= \pm 1, \pm I$, we have

$$
1-\left(\frac{D_{T}}{\pi_{k}}\right)_{4} \frac{1}{\pi_{k}}=\frac{\pi_{k}-\mu}{\pi_{k}}=\frac{\pi_{k}-1+(1-\mu)}{\pi_{k}}, \quad \mu \in\{ \pm 1, \pm I\}
$$

Therefore

$$
v_{2}\left(1-\left(\frac{D_{T}}{\pi_{k}}\right)_{4} \frac{1}{\pi_{k}}\right) \geq \frac{1}{2},
$$

and equality holds if and only if $\left(D_{T} / \pi_{k}\right)_{4}= \pm I$, i.e. $\left(D_{T} / \pi_{k}\right)_{4}^{2}=-1$, that is, $\left[D_{T} / \pi_{k}\right]_{2}=1$. Thus

$$
v_{2}\left(1-\left(\frac{D_{T}}{\pi_{k}}\right)_{4} \frac{1}{\pi_{k}}\right)=\frac{1}{2} \quad \text { if and only if }\left[\frac{D_{T}}{\pi_{k}}\right]_{2}=1 .
$$

By the proof of the first part of the theorem we know that

$$
v_{2}\left(L\left(\bar{\psi}_{D_{T}}, 1\right) / \omega\right) \geq(t(T)-1) / 2, \quad t(T)=\sharp T,
$$

and by our inductive assumption, equality holds if and only if $\delta_{t}\left(D_{T}\right)=1$, $t=t(T)$. Thus

$$
v_{2}\left(\frac{D}{\omega} \overline{\left(\frac{\theta}{D_{T}}\right)_{4}} L_{S}\left(\bar{\psi}_{D_{T}}, 1\right)\right) \geq \frac{t(T)-1}{2}+\frac{n-t(T)}{2}=\frac{n-1}{2},
$$

and equality holds if and only if $\left[D_{T} / \pi_{k}\right]_{2}=1$ (for any $\pi_{k} \mid \widehat{D}_{T}$ ) and $\delta_{t}\left(D_{T}\right.$ ) $=1$. That is to say,

$$
v_{2}\left(\frac{D}{\omega} \overline{\left(\frac{\theta}{D_{T}}\right)_{4}} L_{S}\left(\bar{\psi}_{D_{T}}, 1\right)\right)=\frac{n-1}{2}
$$

if and only if

$$
\left(\prod_{\pi_{k} \mid \widehat{D}_{T}}\left[\frac{D_{T}}{\pi_{k}}\right]_{2}\right) \delta_{t}\left(D_{T}\right)=1 .
$$

For the elliptic curve $E_{D_{T}}: y^{2}=x^{3}-D_{T} x$ and Hecke characters $\psi_{D_{T}}$, by $[\mathrm{Ru} 1,2]$ we know that $L\left(\bar{\psi}_{D_{T}}, 1\right) / \Omega \in K=\mathbb{Q}(I)$, and also we have $\Omega=\omega / \sqrt[4]{D_{T}}$, so

$$
\begin{aligned}
L\left(\bar{\psi}_{D_{T}}, 1\right) / \omega & =\left(\sqrt[4]{D_{T}}\right)^{-1} \cdot L\left(\bar{\psi}_{D_{T}}, 1\right) / \frac{\omega}{\sqrt[4]{D_{T}}} \\
& =\left(\sqrt[4]{D_{T}}\right)^{-1} \cdot L\left(\bar{\psi}_{D_{T}}, 1\right) / \Omega \in K\left(\sqrt[4]{D_{T}}\right),
\end{aligned}
$$

i.e. $L\left(\bar{\psi}_{D_{T}}, 1\right) / \omega \in K\left(\sqrt[4]{D_{T}}\right)$. Thus by Lemma 2.1 we get

$$
\begin{aligned}
& \frac{D}{\overline{\left(\frac{\theta}{D_{T}}\right)_{4}}} L_{S}\left(\bar{\psi}_{D_{T}}, 1\right) \\
& =D \overline{\left(\frac{\theta}{D_{T}}\right)_{4}} \prod_{\pi_{k} \mid \widehat{D}_{T}}\left(1-\left(\frac{D_{T}}{\pi_{k}}\right)_{4} \frac{1}{\pi_{k}}\right) \cdot L\left(\bar{\psi}_{D_{T}}, 1\right) / \omega \in K\left(\sqrt[4]{D_{T}}\right),
\end{aligned}
$$


and if

$$
v_{2}\left(\frac{D}{\omega} \overline{\left(\frac{\theta}{D_{T}}\right)_{4}} L_{S}\left(\bar{\psi}_{D_{T}}, 1\right)\right)=\frac{n-1}{2}
$$

then

$$
\frac{D}{\omega} \overline{\left(\frac{\theta}{D_{T}}\right)_{4}} L_{S}\left(\bar{\psi}_{D_{T}}, 1\right)=(1+I)^{n-1} \alpha_{T} \sqrt[4]{D_{T}^{3}}
$$

where $\alpha_{T} \in K$, and $v_{2}\left(\alpha_{T}\right)=0$ (since $\left.v_{2}\left(\sqrt[4]{D_{T}^{3}}\right)=\frac{3}{4} v_{2}\left(D_{T}\right)=0\right)$. For any subsets $T$ and $T^{\prime}$ of $\{1, \ldots, n\}$, if $v_{2}\left(\alpha_{T}\right)=v_{2}\left(\alpha_{T^{\prime}}\right)=0$, then it can be easily verified that

$$
v_{2}\left(\alpha_{T} \sqrt[4]{D_{T}^{3}}+\alpha_{T^{\prime}} \sqrt[4]{D_{T^{\prime}}^{3}}\right)>0
$$

Thus, consider the terms in the sum

$$
\sum_{\emptyset \neq T \varsubsetneqq\{1, \ldots, n\}} \frac{D}{\omega} \overline{\left(\frac{\theta}{D_{T}}\right)_{4}} L_{S}\left(\bar{\psi}_{D_{T}}, 1\right) .
$$

For any two terms with 2 -adic valuations equal to $(n-1) / 2$, the 2 -adic valuation of their sum is greater than $(n-1) / 2$. Also when $n>1$ we have

$$
v_{2}\left(\frac{D}{\omega} \overline{\left(\frac{\theta}{D_{\emptyset}}\right)_{4}} L_{S}\left(\bar{\psi}_{D_{\emptyset}}, 1\right)\right) \geq 2 n-2 \geq n>\frac{n-1}{2} .
$$

Hence $v_{2}\left(L\left(\bar{\psi}_{D}, 1\right) / \omega\right)=(n-1) / 2$ if and only if one of the following statements is true:

(1) when $v_{2}\left(S^{*}(D)\right)>(n-1) / 2$, in the above sum (2.10), the number of terms with 2 -adic valuation $(n-1) / 2$ is odd;

(2) when $v_{2}\left(S^{*}(D)\right)=(n-1) / 2$, in the above sum $(2.10)$, the number of terms with 2 -adic valuation $(n-1) / 2$ is even.

Statement (1) above means: if $\varepsilon_{n}(D)=0$, then

$$
\begin{array}{r}
\sharp\left\{\neq T \varsubsetneqq\{1, \ldots, n\}: v_{2}\left(\frac{D}{\omega} \overline{\left(\frac{\theta}{D_{T}}\right)_{4}} L_{S}\left(\bar{\psi}_{D_{T}}, 1\right)\right)=\frac{n-1}{2}\right\} \\
=\sharp\left\{\emptyset \neq T \varsubsetneqq\{1, \ldots, n\}:\left(\prod_{\pi_{k} \mid \widehat{D}_{T}}\left[\frac{D_{T}}{\pi_{k}}\right]_{2}\right) \delta_{t}\left(D_{T}\right)=1\right\} \\
\equiv \sum_{\emptyset \neq T \varsubsetneqq\{1, \ldots, n\}}\left(\prod_{\pi_{k} \mid \widehat{D}_{T}}\left[\frac{D_{T}}{\pi_{k}}\right]_{2}\right) \delta_{t}\left(D_{T}\right) \equiv 1(\bmod 2), \\
\delta_{n}(D)=\varepsilon_{n}(D)+\sum_{\emptyset \neq T \varsubsetneqq\{1, \ldots, n\}}\left(\prod_{\pi_{k} \mid \widehat{D}_{T}}\left[\frac{D_{T}}{\pi_{k}}\right]_{2}\right) \delta_{t}\left(D_{T}\right) \equiv 1(\bmod 2) .
\end{array}
$$


And (2) means: if $\varepsilon_{n}(D)=1$ then

$$
\sum_{\emptyset \neq T \mp\{1, \ldots, n\}}\left(\prod_{\pi_{k} \mid \widehat{D}_{T}}\left[\frac{D_{T}}{\pi_{k}}\right]_{2}\right) \delta_{t}\left(D_{T}\right) \equiv 0(\bmod 2),
$$

SO

$$
\begin{aligned}
\delta_{n}(D) & =\varepsilon_{n}(D)+\sum_{\emptyset \neq T \mp\{1, \ldots, n\}}\left(\prod_{\pi_{k} \mid \widehat{D}_{T}}\left[\frac{D_{T}}{\pi_{k}}\right]_{2}\right) \delta_{t}\left(D_{T}\right) \\
& \equiv 1+0 \equiv 1(\bmod 2) .
\end{aligned}
$$

Hence $v_{2}\left(L\left(\bar{\psi}_{D}, 1\right) / \omega\right)=(n-1) / 2$ if and only if $\delta_{n}(D)=1$. This proves the theorem.

Proof of Theorem 3. This theorem follows from Theorem 2 and the main result of Coates-Wiles in [Co-Wi].

For the elliptic curve $E_{D}: y^{2}=x^{3}-D x$ with $D=\pi_{1}^{2} \ldots \pi_{r}^{2} \pi_{r+1} \ldots \pi_{n}$, where $\pi_{k} \equiv 1(\bmod 4)$ are distinct Gaussian prime integers $(k=1, \ldots, n)$, we could prove Theorems 4 and 5 similarly to Theorems 1 and 2 .

\section{References}

[Bir-Ste] B. J. Birch and N. M. Stephens, The parity of the rank of the Mordell-Weil group, Topology 5 (1966), 295-299.

[B-SD] B. J. Birch and H. P. F. Swinnerton-Dyer, Notes on elliptic curves II, J. Reine Angew. Math. 218 (1965), 79-108.

[Co-Wi] J. Coates and A. Wiles, On the conjecture of Birch and Swinnerton-Dyer, Invent. Math. 39 (1977), 223-251.

[Go-Sch] C. Goldstein et N. Schappacher, Séries d'Eisenstein et fonction L de courbes ellipliques à multiplication complexe, J. Reine Angew. Math. 327 (1981), 184218.

[Ire-Ro] K. Ireland and M. Rosen, A Classical Introduction to Modern Number Theory, Grad. Texts in Math. 84, Springer, New York, 1990.

[Raz] M. Razar, The nonvanishing of L(1) for certain elliptic curves with no first descent, Amer. J. Math. 96 (1974), 104-126.

[Ru1] K. Rubin, Tate-Shafarevich groups and L-functions of elliptic curves with complex multiplication, Invent. Math. 89 (1987), 527-560.

[Ru2] - The "main conjectures" of Iwasawa theory for imaginary quadratic fields, ibid. 103 (1991), 25-68.

[Sil1] J. H. Silverman, The Arithmetic of Elliptic Curves, Grad. Texts in Math. 106, Springer, New York, 1986.

[Sil2] - Advanced Topics in the Arithmetic of Elliptic Curves, Grad. Texts in Math. 151, Springer, 1994.

[Tun] J. B. Tunnell, A classical Diophantine problem and modular forms of weight $\frac{3}{2}$, Invent. Math. 72 (1983), 323-334.

[We] A. Weil, Elliptic Functions According to Eisenstein and Kronecker, Springer, 1976. 
[Zhao] C. L. Zhao, A criterion for elliptic curves with lowest 2-power in L(1), Math. Proc. Cambridge Philos. Soc. 121 (1997), 385-400.

Center for Advanced Study

Tsinghua University

Beijing 100084, P.R. China

E-mail: derong@castu.tsinghua.edu.cn
Department of Mathematical Sciences

Tsinghua University Beijing 100084, P.R. China

E-mail: xianke@tsinghua.edu.cn

Received on 12.4.2001

and in revised form on 14.7.2001 